\title{
Total Hip Arthroplasty for Periacetabular Metastatic Disease: An Original Technique of Reconstruction According to Harrington Classification
}

Julien Wegrzyn 1, 2, 3, Matthieu Malatray 1, Vincent Pibarot 1, Cyrille Confavreux 2, 3, 4

\author{
1. Department of Orthopedic Surgery - Pavillon T, Hôpital Edouard Herriot, Hospices Civils de Lyon, France, 2. INSERM UMR 1033, Université de Lyon, France, \\ 3. Centre Expert des Métastases Osseuses, Hospices Civils de Lyon, France, 4. Department of Rheumatology, Centre Hospitalier Lyon Sud, Hospices Civils de Lyon, France
}

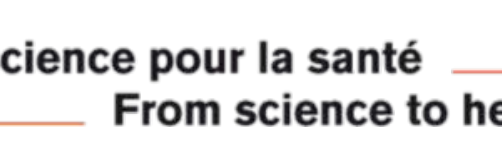

\section{INTRODUCTION}

Periacetabular metastatic disease requires complex acetabular reconstruction with high risks of complication in very frail patients. Various techniques have been proposed consisting in cement-rebar reinforced hip reconstruction to allow cemented total hip arthroplasty (THA) [1]. However, the optimal technique has not yet being defined. To our knowledge, the current study reported the largest prospective cohort of patients with periacetabular metastatic disease undergoing an original technique of acetabular reconstruction combining bone metastasis curettage and cementation, and dual mobility cup cemented into an acetabular reinforcement device according to the Harrington classification.

This study aimed to demonstrate that this technique:

$1 /$ was effective for reconstructing periacetabular metastatic bone defects with minimal risk of complication,

$2 /$ restored the ability to ambulate with immediate full weight-bearing allowing these patients to be functionally independent in the community,

3 / achieved significant pain relief with decreased consumption of narcotic analgesics,

4/ and provided durable reconstruction that was likely to face to adjuvant radiation therapy and mechanical solicitations for long survivors.

\section{METHODS}

A continuous series of 131 THA performed in 126 patients with periacetabular metastatic disease (mean age $=64 \pm 13$ years) was prospectively included in this study. IRB approval and patient's informed consent were obtained.

Metastatic acetabular bone defect was graded according to the Harrington classification with 19 THA (15\%) performed for Harrington class I defect, 63 THA (48\%) for Harrington class II defect and 49 THA (37\%) for Harrington class III defect [1]. Combined acetabular and proximal femoral bone metastasis was diagnosed in 51 THA (39\%) involving the femoral head and neck in 25 cases $(19 \%)$, the intertrochanteric part of the femur in 15 cases (11\%), and the subtrochanteric part of the femur in 11 cases (8\%).

After bone metastasis curettage and cementation, an original technique of acetabular reconstruction was performed using a dual mobility cup cemented into an acetabular reinforcement device (i.e.; Kerboull cross-plate or Burch-Schneider anti-protrusio cage) according to the Harrington classification (Figure). On the femoral side, the femoral reconstruction was performed using a proximal femoral resection arthroplasty with preservation of the abductor mechanism continuity in cases of combined intertrochanteric or subtrochanteric femoral metastasis. All the other cases were managed with a conventional non-modular cemented femoral stem.

Functional outcome for independent ambulation in the community, pain relief, and occurrence of dislocation or mechanical failure of the acetabular reconstruction were assessed.

\section{RESULTS}

The mean follow-up of the entire series was $33 \pm 17$ months. One patient $(0.8 \%)$ deceased intraoperatively due to a bone cement implantation syndrome during the cementation of a proximal femoral arthroplasty and 2 patients (2\%) deceased within the first postoperative month. The pre- to post-operative functional outcome for walking and functioning during daily living activities improved significantly $(p<0.001)$ (Table). The pain relief as determined by the pre- to post-operative comparison of narcotic analgesics consumption was significant $(p<0.001)$ (Table). The dislocation rate was $2 \%$. Two of the three cases of dislocation occurred in acetabular reconstructions associated with proximal femoral arthroplasty. No mechanical failure or aseptic loosening of the acetabular reconstruction was observed despite a case of local progression of the metastatic disease in a radio-resistant kidney carcinoma.

\begin{tabular}{|c|c|c|c|}
\hline & & $\begin{array}{l}\text { Preoperative } \\
\text { (131 hips) }\end{array}$ & $\begin{array}{l}\text { Postoperative } \\
\text { (128 hips) }\end{array}$ \\
\hline \multirow{3}{*}{ 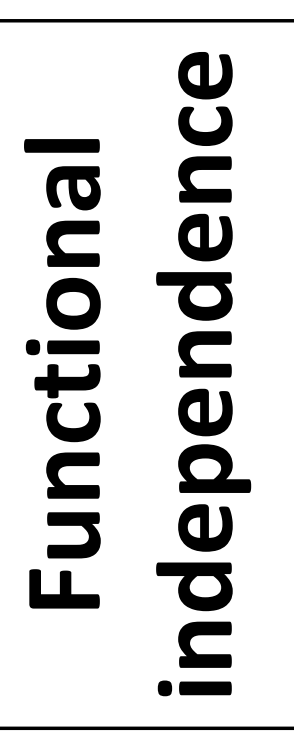 } & Not able to walk or use a walker only in their home & $99(76 \%)$ & $2(2 \%)$ \\
\hline & Walk with 2 crutches for a distance $<30$ minutes & $20(15 \%)$ & $11(8 \%)$ \\
\hline & Walk with 1 crutch or without for a distance $>30$ minutes & $12(9 \%)$ & $115(90 \%)$ \\
\hline \multirow{4}{*}{ 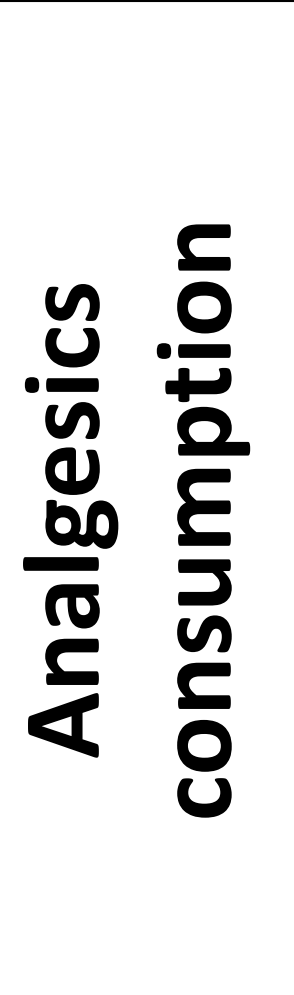 } & $\begin{array}{l}\text { Severe hip pain requiring continuous use } \\
\text { of narcotic analgesics }\end{array}$ & $122(93 \%)$ & $4(3 \%)$ \\
\hline & $\begin{array}{c}\text { Moderate hip pain requiring occasional use } \\
\text { of narcotic analgesics }\end{array}$ & $9(7 \%)$ & $18(14 \%)$ \\
\hline & Mild hip pain requiring use of non-narcotic drugs & $0(0 \%)$ & $81(63 \%)$ \\
\hline & Light or no pain with occasional use of acetaminophen & $0(0 \%)$ & $25(20 \%)$ \\
\hline
\end{tabular}

Table: Pre- to post-operative comparisons of function and pain level (n THA, (\%)).
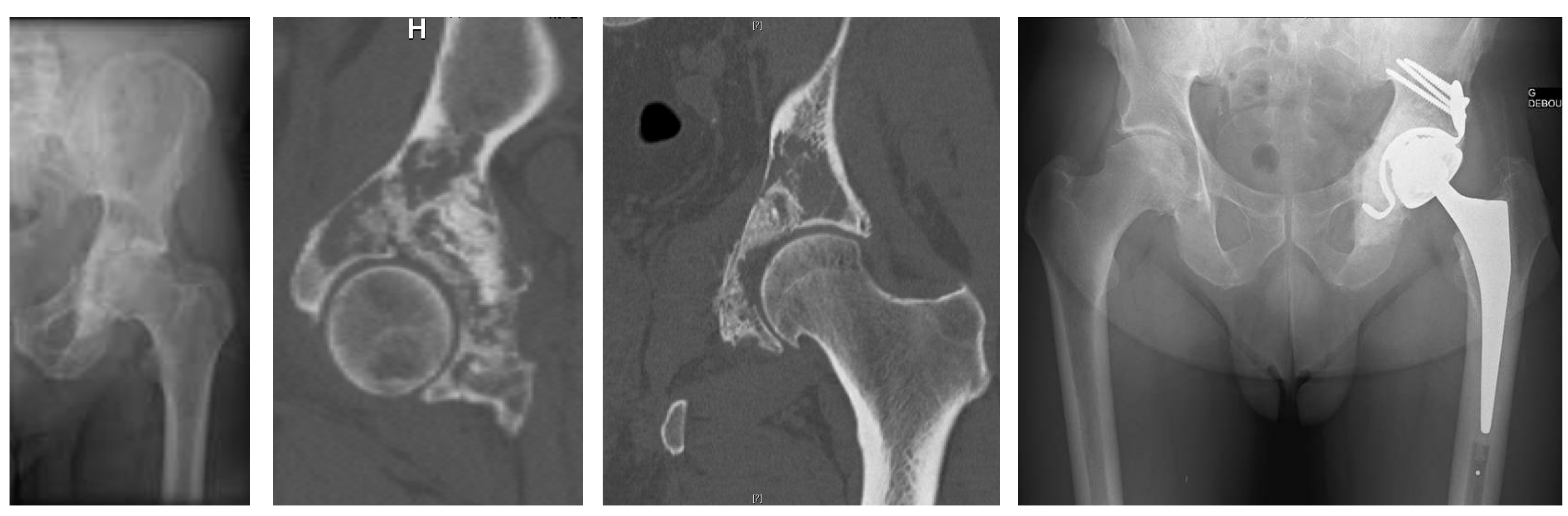

Figure: Acetabular reconstruction using a Kerboull cross-plate and a cemented dual mobility cup in a case of Harrington class III metastatic bone defect related to a prostate carcinoma.

\section{DISCUSSION}

This study emphasized that our original technique based on a Harrington classification algorithm and combining bone metastasis curettage and cementation, and dual mobility cup cemented into a Kerboull cross-plate or Burch-Schneider anti-protrusio cage was effective to restore painless functional independence with immediate full weight-bearing ambulation [2].

This technique ensured a durable acetabular reconstruction able to face to adjuvant radiation therapy and mechanical solicitation for long survivors [2]

In addition, the use of dual mobility cup limited the risk of dislocation in patients undergoing THA for periacetabular metastatic disease [2].

\section{CLINICAL RELEVANCE}

This study aimed to address the challenge of how to best restore painless independent ambulation in the community and provide a durable acetabular reconstruction lasting the lifespan of metastatic patients with minimal risk of complication and prevention of THA dislocation [2]

\section{References:}

[1] Harrington KD. J Bone Joint Surg Am 1981, [2] Wegrzyn J et al. J Arthroplasty 2018 